PROCEEDINGS OF THE

AMERICAN MATHEMATICAL SOCIETY

Volume 132, Number 3, Pages 781-782

S 0002-9939(03)07111-9

Article electronically published on October 2, 2003

\title{
A REMARK TO A THEOREM OF YU. A. ABRAMOVICH
}

\author{
EDUARD YU. EMEL'YANOV
}

(Communicated by Joseph A. Ball)

\begin{abstract}
A remarkable theorem due to Abramovich (1988) states that any surjective positive isometry on a Banach lattice has a positive inverse. In this note we discuss a renorming problem for Banach lattices and show that the theorem cannot be generalized to the case of the doubly power bounded positive operators.
\end{abstract}

Positive isometries of Banach lattices possess many attractive properties. For instance, due to the well-known result of J. Lamperti [2, thm. 3.1], positive isometries on $L_{p}$-spaces $(1 \leq p \leq \infty, p \neq 2)$ have representations as weighted shift operators with positive weight. However, it can be that we have an operator before one of equivalent norms on the Banach lattice is fixed. How does one determine whether the operator is a positive invertible isometry with respect to some appropriate equivalent lattice norm? In the Banach space setting such a question is trivial, namely, the necessary and sufficient condition for an operator $T$ in a Banach space $X$ to be an invertible isometry with respect to some equivalent norm is that $T$ be doubly power bounded, i.e., $\sup \left\{\left\|T^{n}\right\|: n \in \mathbb{Z}\right\}<\infty$; then an equivalent norm $\|\cdot\|_{T}$ under which $T$ and $T^{-1}$ are isometries can be defined as

$$
\|x\|_{T}:=\sup \left\{\left\|T^{n} x\right\|: n \in \mathbb{Z}\right\} \quad(\forall x \in X) .
$$

However, if we consider a positive operator on a Banach lattice, this is not a Banach lattice norm, since we do not know if $T^{-1} \geq 0$. Whenever this occurs, the operator $T$ is a lattice automorphism and the preceding formula really defines an equivalent Banach lattice norm. Due to Abramovich's theorem [1, thm. 1] this occurs if $T$ is a positive invertible isometry. On the other hand, it is well known that every doubly power bounded positive operator on a finite-dimensional Banach lattice has a positive inverse. The discussion above make the following question substantial. Is $T^{-1}$ positive for any doubly power bounded positive operator on a Banach lattice? We will show that the answer in general is negative.

Theorem 1. Given $\omega \notin \mathbb{R}$, take $\Omega=\mathbb{R} \cup\{\omega\}$ and let a measure $\mu$ on the Borel algebra $\mathcal{B}=\mathcal{B}(\Omega)$ be defined as the Lebesgue measure on $\mathcal{B}(\mathbb{R})$ and $\mu(\{\omega\})=1$.

Then for any $\varepsilon>0$ there exists a positive operator on $L_{1}(\Omega, \mathcal{B}, \mu)$ with nonpositive inverse that satisfies $\sup _{n \in \mathbb{Z}}\left\|T^{n}\right\| \leq 1+\varepsilon$.

Received by the editors June 19, 2002 and, in revised form, October 25, 2002.

2000 Mathematics Subject Classification. Primary 47B65, 46B03, 46B42.

Key words and phrases. Positive isometry, doubly power bounded operator, renorming problem. 
Proof. Let $\varepsilon>0$. Consider a measure-preserving automorphism $S$ on $\Omega$ defined by $S(\omega)=\omega$ and $S(t)=t-1$ whenever $t \in \mathbb{R}$, and define a positive operator $T$ on $L_{1}(\Omega, \mathcal{B}, \mu)$ by

$$
T f:=f \circ S+\varepsilon \cdot\left[\int_{0}^{1} f d \mu\right] \cdot \chi_{\{\omega\}},
$$

where as usual $\chi_{A}$ is the indicator function of a subset $A$. Then it is easy to see that

$$
\begin{aligned}
T^{n} f & =f \circ S^{n}+\varepsilon \cdot\left[\sum_{i=1}^{n} \int_{0}^{1} f \circ S^{i-1} d \mu\right] \cdot \chi_{\{\omega\}} \\
& =f \circ S^{n}+\varepsilon \cdot\left[\sum_{i=1}^{n} \int_{n-i}^{n-i+1} f \circ S^{n-1} d \mu\right] \cdot \chi_{\{\omega\}} \\
& =f \circ S^{n}+\varepsilon \cdot\left[\int_{0}^{n} f \circ S^{n-1} d \mu\right] \cdot \chi_{\{\omega\}}
\end{aligned}
$$

for all $n \in \mathbb{Z}_{+}$and $f \in L_{1}(\Omega, \mathcal{B}, \mu)$. A similar computation shows that

$$
T^{n} f=f \circ S^{n}-\varepsilon \cdot\left[\int_{-n}^{0} f \circ S^{n-1} d \mu\right] \cdot \chi_{\{\omega\}}
$$

for all $n \in \mathbb{Z} \backslash \mathbb{Z}_{+}$and $f \in L_{1}(\Omega, \mathcal{B}, \mu)$; in particular, $T^{-1}$ is not positive. Moreover,

$$
\left\|T^{n} f\right\| \leq\left\|f \circ S^{n}\right\|+\varepsilon \cdot\left[\int_{-\infty}^{\infty}\left|f \circ S^{n-1}\right| d \mu\right] \cdot\left\|\chi_{\{\omega\}}\right\|=(1+\varepsilon)\|f\|
$$

for all $n \in \mathbb{Z}$ and $f \in L_{1}(\Omega, \mathcal{B}, \mu)$, which provides the required property $\sup \left\{\left\|T^{n}\right\|\right.$ : $n \in \mathbb{Z}\} \leq 1+\varepsilon$.

Let us give several simple final remarks. First, the adjoint operator $T^{*}$ of $T$ possesses similar properties on $L_{\infty}(\Omega, \mathcal{B}, \mu)$. Second, the operator $T$ constructed in the proof acts in the same manner on $L_{p}(\Omega, \mathcal{B}, \mu)$ for any $1<p \leq \infty$; however, in this case, $\sup \left\{\left\|T^{n}\right\|: n \in \mathbb{Z}\right\}<\infty$ fails in the $L_{p}$-norm. Third, the adjunction of the external point $\omega$ to $\mathbb{R}$ is not essential, and an operator with the same properties may be constructed on $L_{1}(\mathbb{R})$ too, but this leads to complications with the construction.

\section{REFERENCES}

[1] Yu. A. Abramovich, Isometries of normed lattices, (Russian), Optimizatsiya 43(60) (1988), 74-80. MR 90k:46042

[2] J. Lamperti, On the isometries of certain function-spaces, Pacific J. Math. 8 (1958), 459-466. MR 21:3764

Sobolev Institute of Mathematics, Acad. Koptyug PR. 4, 630090 Novosibirsk, Russia

E-mail address: emelanov@math.nsc.ru 